\title{
(6) OPEN ACCESS \\ Victims, vectors and villains: are those who opt out of vaccination morally responsible for the deaths of others?
}

\author{
Euzebiusz Jamrozik, ${ }^{1}$ Toby Handfield, ${ }^{2}$ Michael J Selgelid ${ }^{1}$
}

${ }^{1}$ Centre for Human Bioethics, SOPHIS, Monash University, Clayton, Victoria, Australia ${ }^{2}$ Department of Philosophy, SOPHIS, Monash University, Clayton, Victoria, Australia

\section{Correspondence to}

Dr Euzebiusz Jamrozik, Centre for Human Bioethics, Monash University, Clayton, VIC, 3800, Australia;

zeb.jamrozik@monash.edu

Received 17 December 2015 Revised 22 August 2016 Accepted 6 September 2016 Published Online First 3 October 2016
CrossMark

To cite: Jamrozik $E$, Handfield T, Selgelid MJ. J Med Ethics 2016;42:762768.

\section{ABSTRACT}

Mass vaccination has been a successful public health strategy for many contagious diseases. The immunity of the vaccinated also protects others who cannot be safely or effectively vaccinated-including infants and the immunosuppressed. When vaccination rates fall, diseases like measles can rapidly resurge in a population. Those who cannot be vaccinated for medical reasons are at the highest risk of severe disease and death. They thus may bear the burden of others' freedom to opt out of vaccination. It is often asked whether it is legitimate for states to adopt and enforce mandatory universal vaccination. Yet this neglects a related question: are those who opt out, where it is permitted, morally responsible when others are harmed or die as a result of their decision? In this article, we argue that individuals who opt out of vaccination are morally responsible for resultant harms to others. Using measles as our main example, we demonstrate the ways in which opting out of vaccination can result in a significant risk of harm and death to others, especially infants and the immunosuppressed. We argue that imposing these risks without good justification is blameworthy and examine ways of reaching a coherent understanding of individual moral responsibility for harms in the context of the collective action required for disease transmission. Finally, we consider several objections to this view, provide counterarguments and suggest morally permissible alternatives to mandatory universal vaccination including controlled infection, self-imposed social isolation and financial penalties for refusal to vaccinate.

\section{INTRODUCTION}

\section{Vaccine-preventable infectious diseases}

Many vaccine-preventable infections are transmitted between human beings and can cause serious harm or death. Measles, for example, is one of the most infectious known human viruses. It is transmitted via the airborne route, and outbreaks can result from minimal social contact between contagious and susceptible individuals. ${ }^{1} 2$ In most people, measles causes mild disease, but in some cases there are serious sequelae including lung inflammation (pneumonitis), brain inflammation (encephalitis), permanent disability and death. US historical data suggest a death rate of about 1 in every 350-1200 cases. ${ }^{13}$ Risks of both complications and death are highest in infants, young children, the malnourished and the immunosuppressed. ${ }^{3}$ Prior to widespread measles vaccination, there were, on average, $>500000$ cases of measles and 400 deaths per year in the USA. ${ }^{1}$ Measles previously also accounted for a significant proportion of deaths among young people undergoing chemotherapy for leukaemia ${ }^{i} .^{4}$ Currently, measles continues to cause $>100000$ deaths per year worldwide-mostly in children under 5 years of age in sub-Saharan Africa and India, in communities where vaccination rates remain suboptimal. $^{5}$

Modern inoculations for many common diseases are extremely safe and effectively prevent illness in the vast majority of those vaccinated. Vaccines have played a major role in the eradication of smallpox and near eradication of polio. High rates of vaccination against such diseases also protect those who cannot be safely vaccinated, such as infants and the immunosuppressed, through promotion of herd protection (or 'herd immunity'). ${ }^{6}$

The measles vaccine is especially safe and effective, with significant side effects from vaccination being exceedingly rare. A careful long-term follow-up study of measles, mumps, and rubella (MMR) vaccination found zero deaths due to the vaccine and a less than one in a million chance of encephalitis. ${ }^{7}$

There are several groups that cannot be safely or effectively vaccinated with some vaccines (eg, infants, the immunodeficient and the immunosuppressed). ${ }^{8}{ }^{9}$ The only way these vulnerable individuals can be effectively protected against common infections, apart from extremely onerous social isolation (eg, 'boy in a bubble' scenarios), is through herd protection (achieved by high vaccination rates). ${ }^{\text {ii }}$ Herd protection models for measles suggest that well over $90 \%$ of a population need to be vaccinated in order to reliably prevent sustained transmission. ${ }^{10-12}$ Recent outbreaks have shown that measles can spread quickly and cause significant harm when vaccination rates fall. ${ }^{2}{ }^{3}$ The presence of such vulnerable individuals raises questions as to whether these people should bear the burdens of the decisions of others not to vaccinate and to what degree the latter should be considered morally responsible for resultant harms to the former.

In this article, we propose that those who opt out of vaccination are morally responsible (and in particular, blameworthy) for the harms suffered by others as a result of infectious outbreaks. In other words, non-vaccinators are morally blameworthy for the morbidity and mortality caused by

${ }^{\mathrm{i}}$ Measles still poses significant risks in this context despite prior vaccination of patients with cancer.

${ }^{\mathrm{i}}$ That is, a sufficiently high proportion of those with whom they come into contact must be vaccinated or immune from past infection: to minimise risk, this proportion must approach $100 \%$. 
infectious diseases that can be prevented by vaccination. Exactly how blame should in practice be apportioned among different individuals is a question that is beyond the scope of this article to resolve: but we show that there is at least one plausible and morally defensible way that blame could be ascribed to non-vaccinators. If our argument is sound, it joins other significant grounds that support public health intervention in this area. ${ }^{13-16}$

We focus on the example of measles because it is highly contagious in social situations where people merely share the same air. Similar considerations apply to many other common infections transmitted in similar ways, but we leave aside diseases with other modes of transmission, noting that these differences and their moral aspects have received some attention elsewhere. ${ }^{16} 17$

One way to support our thesis would be to analyse the concept of moral responsibility so as to identify sufficient conditions, and then to show that these conditions apply to non-vaccinators and the harms they cause. While we think this route is defensible, it is difficult to frame in a way that is not question-begging. Any account of sufficient conditions (for moral responsibility) that supports our conclusion is likely to be more contentious than the conclusion itself.

Instead, we adopt the following, two-pronged strategy. First, we simply conjecture that non-vaccinators are responsible for harms and present some relatively uncontentious necessary conditions of moral responsibility. We consider some possible objections to our responsibility thesis, organised around these conditions, and show that they all fail. Thus, we show that there is no knockdown objection to our claim. Second, we argue that there are a number of cases where we do in fact consider individuals blameworthy for harms that bear important similarities to the harms caused by non-vaccinators. The legitimacy of considering individuals responsible in these other cases suggests that it is analogously legitimate to consider non-vaccinators responsible.

\section{MORAL RESPONSIBILITY, BLAME AND RISK}

Those who are morally responsible for an outcome are proper subjects of praise or blame for that outcome. So, for our purposes, to say that someone is morally responsible for an outcome $\mathrm{O}$ is equivalent to saying that they are worthy of (ie, deserve) praise or blame for $\mathrm{O}$. The project of analysing the necessary and sufficient conditions for moral responsibility, however, remains contentious. We do not hope to offer a complete account here. Instead, we propose three necessary conditions for moral responsibility. The conditions will be specified only roughly, but with enough precision to ensure that they can be uncontroversially applied to the vast majority of cases of harm caused by refusal to vaccinate.

An agent $\mathrm{A}$ is morally responsible for outcome $\mathrm{O}$ only if

- (Cause) Agent A performs an action $\Phi$ that is a relevant part of a causal explanation of $\mathrm{O}$.

- (Salience) Outcome O is morally salient-it is a good or bad outcome.

- (Foreseeability) Outcome $\mathrm{O}$ is a predictable or foreseeable outcome of action $\Phi$.

Evidently, refusal to vaccinate frequently satisfies the salience condition: the harms caused by infectious diseases are highly significant. Further, we see no difficulty meeting the foreseeability condition. No doubt, when inoculation was a novel invention and mechanisms of transmission were less widely understood, refusal of a vaccination would not have always satisfied the foreseeability condition. But now, at least in those countries where vaccination is widely available, it is practically common knowledge that non-vaccination can have dangerous consequences for oneself and for others. The most serious source of potential resistance is the causal condition. Although it is undeniable that some individuals' refusal to vaccinate is part of the causal explanation of some infections, it might be disputed whether the 'relevant' sort of causal connection required for moral responsibility is present. For the moment, we put this objection to the side: it will be addressed in a section below.

These conditions provide a natural way to identify possible sources of objection to our conjecture that non-vaccinators are responsible for harms that result from their resultant lack of immunity. In the following sections, we consider and address the following purported objections:

1. Non-vaccinators lack the relevant sort of agency to be considered responsible for harms that result from a failure to vaccinate. The harms do not arise from anything that nonvaccinators $d o$.

2. Harms due to a failure to vaccinate are due to collective behaviours of many individuals, and consequently the causal condition for responsibility is not satisfied.

3. Non-vaccinators may be morally responsible for the decision whether or not to vaccinate, but there is no duty to vaccinate. Vaccination is supererogatory, and so failure to vaccinate is not blameworthy.

\section{OBJECTION: NON-VACCINATION AND AGENCY}

To legitimately consider someone morally responsible for an outcome, it is necessary that the person had a relevant form of agency, and that this agency was involved in the appropriate way in the outcome for which they are attributed responsibility. It is for this sort of reason that it does not make much sense to consider someone responsible for the fact that 2 is a prime number; nor for the actions of their ancestors of 20 generations earlier. These states of affairs do not stand in the appropriate causal relationship to the agency of an allegedly responsible person. No one can causally influence what happened 20 generations earlier, nor can one influence a mathematical fact.

In non-vaccination cases, it might be objected that the harms that result from non-vaccination are not harms that the nonvaccinator has caused to occur; they are, rather, harms that the non-vaccinator has merely failed to prevent. Or a somewhat different claim might be made: that the agent has not intended the harm. For one or both of these reasons, it might be argued, the agency of the non-vaccinator is not sufficiently involved in the harm for us to hold the agent responsible.

Considered generally, this type of objection relies on two claims. First: a conceptual claim is required, distinguishing two types of harm, such that stereotypical wrongful harms fall in one category, while harms suffered as a causal consequence of non-vaccination fall in the other. As already suggested, all manner of distinctions might be used in this way: we can distinguish between harms that are a result of doing versus allowing; or harms that we commit rather than those that we omit to prevent; or harms that we intend versus those that we do not intend. ${ }^{18}$ Call this first part of the response the conceptual claim.

Second, it must be argued that the distinction identified in the conceptual claim separates states of affairs for which we may be held responsible and those for which we cannot. "We can be held responsible for the harms that we inflict on others, but not for those that we merely fail to prevent", or something to this effect. Call this the moral significance claim. 
This response is implausible because there are numerous examples-unrelated to vaccination-which illustrate the credibility of considering individuals responsible for harms that they both (i) did not intend and (ii) did not cause directly by their actions, but merely failed to prevent. Consider:

Intoxicated driver. A driver is driving while intoxicated at a safe and reasonable speed. Due to her intoxication, however, she blacks out briefly. During the blackout, she runs a red light, and her vehicle collides with a pedestrian, killing him.

In such cases, the relevant agent does not intend the harm: the harm comes about as a result of failures to act. Indeed, at the crucial time of the red light signal, the driver is not capable of acting in the required way because she is unconscious. Perhaps in some cultures, and in some historical periods, this driver would not be considered responsible for her careless behaviour; but in many cultures and jurisdictions she would be, and it is not credible to suppose that some conceptual error is occurring in such cases. If it is coherent to consider individuals responsible in cases like this, then there is no principled basis for objection that non-vaccinators cannot be considered responsible because they lack appropriate agency for the harms that they transmit to others. That said, it remains very plausible that intentionally caused harms warrant more blame than unintentionally caused harms. We might think that the person who accidentally forgets to get vaccinated deserves less blame than the person who actively decides against vaccination. In addition, in some countries vaccination is difficult to obtain, and where it is not universally and freely available (or heavily subsidised), some people may have difficulty paying the financial costs. Cases like these would seem to be much less culpable, especially where vaccines are not available, and blame may be attributable to local or international public health agencies. But this is no objection to our thesis that non-vaccinators (with easy access to vaccines) can legitimately be considered responsible.

\section{OBJECTION: COLLECTIVE ACTION AND INDIVIDUAL RESPONSIBILITY}

Typically, one person being unvaccinated is extremely unlikely to cause harm to others because the probability of having direct contact with both a contagious person (to become infected) and a vulnerable person (infecting them) is low, at least in populations where vaccine uptake levels are high. Actual transmissions to vulnerable individuals will typically involve a chain of transmission involving dozens of unvaccinated individuals.

Is the collective nature of the harm-causing process compatible with considering individuals responsible? We might have concerns about overdetermination (where multiple chains of transmission within an outbreak are sufficient to cause a given harm) or because of more general concerns that our ordinary assumptions about individual responsibility are not appropriate to collective causation.

To address the concern about overdetermination first: this will depend to a large extent on disease epidemiology. Take two extreme cases: ${ }^{\text {iii }}$ (i) endemic malaria: each sick person's clinical case of malaria is likely to be overdetermined since they will be infected with parasites transmitted to them by many other people, and each episode of transmission is itself potentially sufficient to cause clinical malaria; and (ii) measles in a country with a high vaccination rate: here each sick person's clinical case of measles is much more likely to have been transmitted to

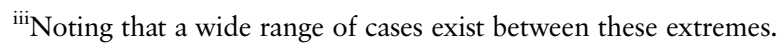

them by just one other person, and each sick person is unlikely to have had sufficient contact with more than one infectious case. $^{\text {iv }}$

In cases like the former (endemic malaria), our ordinary ascriptions of responsibility might seem inappropriate. Blaming someone for a bad outcome usually implies that, had the blamed party behaved otherwise, the bad outcome would not have occurred. This is precisely what fails to hold in overdetermination cases.

Legal doctrine has evolved ingenious strategies to address this sort of concern so that parties who participate in multiple, simultaneous wrongs (eg, Jones burns down the factory from the outside, Smith blows it up from the inside; had one of them not acted, the factory would have been destroyed anyway) cannot benefit from the overdetermination of the damage they collectively do. In dealing with overdetermination of risks, the measures become even more conceptually sophisticated. ${ }^{19} 20$ We suggest something similar should happen here: we should adjust our understanding of causation so as to ensure that overdetermined harms are still caused in the relevant sense. Blind adherence to a counterfactual test of causal responsibility will give an absurd answer to questions of blameworthiness. That suggests we should reject the counterfactual test rather than denying that anyone should be blamed for overdetermined harms. ${ }^{\mathrm{v}}$

Overdetermination aside, the question of how to apportion individual responsibility in cases where one group (those who opt out of vaccination) collectively causes harms to another group (those who are vulnerable to vaccine-preventable disease) remains. Even for cases like measles in high-income countries, one individual alone opting out of vaccination is rarely sufficient to cause infection in another. Such transmission will most commonly involve a causal chain leading back to many other susceptible people, some of whom have chosen to remain susceptible by opting out of vaccination. ${ }^{\mathrm{vi}}$

Suppose a chain of transmitters (T1 $\rightarrow$ T2 $\rightarrow \cdots \rightarrow$ T100) infect an immunosuppressed victim V. Suppose all T1-T100 opted out of vaccination, knowing that this might cause harm to others. Suppose further that they all engaged in similar riskcreating behaviours: they had similar exposure to immunosuppressed individuals and took similar precautions with washing hands and the like. Had T100 obtained a vaccination, V would not have become unwell. ${ }^{\text {vii }}$ But it is also the case that had T99 obtained a vaccination, V would not have become unwell. And so on, for every member of the chain. So who is responsible?

At this point, it is helpful to stress the distinction between moral responsibility for conduct versus moral responsibility for consequences of our conduct. It is possible to think that

\footnotetext{
${ }^{\text {iv }}$ Although for both malaria and measles there is some evidence of a relationship between pathogen 'dose' and severity of disease.

vEven where overdetermination applies, other authors have provided a coherent account of moral reasons not to contribute to collective harms where those harms are likely to be overdetermined (eg, climate change). Barry C \& Overland G. Individual Responsibility for Carbon Emissions. In: eremy Moss (ed), Climate Change and Justice, Cambridge University Press, Cambridge, UK, 2015:165-183. Similar reasoning may apply to infection, and this may be a fruitful path for future enquiry.

${ }^{v i}$ It is not merely that these events are earlier in time to the ultimate transmission event of interest (eg, to a vulnerable person) but that it is the combined actions (ie, refusals to vaccinate) of the collective involved in the chain that are together sufficient to cause harm.

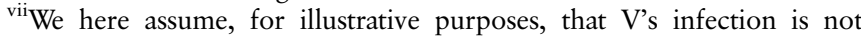
overdetermined-that is, $\mathrm{V}$ would not have been infected by another chain of transmitters if the chain of infection from T1 to T100 to V had not occurred.
} 
someone is blameworthy for their conduct without holding them blameworthy for all the bad consequences of their conduct. The distinction is philosophically important because the outcomes of our actions are never entirely under our control, whereas our conduct is-at least to a much greater degree. If we consider individuals responsible to different degrees because their conduct, though similar, led to different outcomes, then we introduce an element of luck into our system of moral judgement. This 'moral luck' is a pervasive feature of normal moral practices involving praise and blame, but it is controversial among theorists whether it can be justified. ${ }^{21}$

So, in the present case, with respect to their conduct, $e x$ hypothesi, each of the 100 individuals is equally blameworthy. They all imposed similar risks and had similar foreknowledge and control over those risks. Their moral responsibility for harm to others will be affected by moral luck because not everyone who behaves in a similarly blameworthy way will cause the same harms. While all non-vaccinators are guilty of equally blameworthy conduct (ie, they have all behaved equally badly), only those who are actually involved in chains of transmission to vulnerable individuals end up being morally responsible/ blameworthy for harm because causation is a necessary condition for blameworthiness; and the causal difference between these groups is a matter of sheer luck. With regard to moral responsibility for harm, therefore, moral luck is inescapable.

We claim that those non-vaccinators involved in a chain causing harm are blameworthy for avoidable harms arising from non-vaccination and, in practice, this means that they could legitimately be blamed for these harms. ${ }^{\mathrm{viii}}$ We do not claim that there is a uniquely appropriate way, in theory or practice, to assign blame for the eventual harms, at least in part because of the element of moral luck that influences the outcomes of risky behaviours. With regard to theory, there may be multiple plausible views about the degree of blame that the 100 individuals in question deserve for harm to V. With regard to practice, indeterminacy could be resolved by adopting particular conventions or laws. There are numerous examples of stable moral practices in which we hold people blameworthy for the outcomes of their collective risky behaviour and we see no reason to think that an appropriate custom could not be devised for non-vaccination.

To illustrate, consider three ways (not exhaustive) in which we might ascribe blame to those involved in chains of transmission:

i. Nobody among T1-T100 is blamed for the harm to V.

ii. Only T100 is blamed for the harm to V (proximate cause).

iii. All individuals, T1 to T100, are blamed to an equal degree for the harm to $\mathrm{V}$.

With regard to the first option: is it plausible to think that no one is blameworthy for the harm? A crucial requirement for any credible account of individual and collective responsibility is that individuals are not left with perverse incentives to (collectively) impose large risks on others for little or no benefit to themselves. Allowing non-vaccinators to escape all responsibility for subsequent deaths fails this basic test. ${ }^{22}$

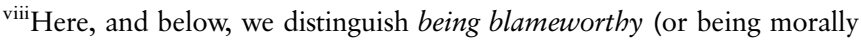
responsible) in theory from being blamed (or being held morally responsible) in practice. While the moral practice of blame might often map onto theoretical accounts of blameworthiness, this need not always be the case. For practical reasons, for example, we may sometimes choose not to actually blame those who are blameworthy (for harm). While the majority of this article focuses on the question of who is morally blameworthy for what (in theory), in what follows we also consider suggestions regarding who should be blamed for what (in practice).
}

The second of these policies (blaming the proximate cause T100) might be appealing in practice because of its convenience: it requires only one individual be found to receive the warranted blame. Theoretically, however, it is implausible to consider the proximate cause more blameworthy (for harm) than any other member of the chain. Of 100 individuals who imposed similar risks and had similar causal involvement in the death, it was sheer luck that made T100 the ultimate transmitter of the harm to a vulnerable person. ${ }^{23}$ There would thus be a very strong element of moral luck, making it, on some views, unfair to blame only T100, given that T1-T99 were all similarly blameworthy. Nevertheless, a moral community could have practical or policy reasons (eg, deterrence) to blame only the proximate transmitter-even if this blame were out of proportion to her causal role in the harm. ${ }^{\text {ix }}$

The third policy would regard each individual in the chain (who had reasonable opportunity to act otherwise) as blameworthy for the harms that arise. By considering each morally responsible, we acknowledge that each is implicated in causing avoidable harm. ${ }^{\mathrm{x}}$

That said, there remain many unresolved questions about how to apportion blame in collective action cases. In our contrived example, the causal roles of T1-T100 are all similar. But in cases where the contributions are heterogeneous (eg, some better foresee harms than others, some take more/better infection control measures than others, etc), we may need to discriminate different degrees of blame deserved by the various agents. For instance, one fundamental issue is whether it is possible to meaningfully quantify the degree of causal contribution that an agent (or chance) makes to an outcome. Intuitively, we can at least make a partial ranking of contributions in multiagent cases: some contribute more than others, though some contributions may be incomparable. While we may take some guidance from this partial ranking information, it will leave some issues indeterminate. For instance, if A makes a greater contribution than $\mathrm{B}$, this may constrain us to ascribe a greater quantum of deserved blame to A. But suppose that a third agent $\mathrm{C}$ is involved, and neither A's contribution nor B's contribution is comparable to the contribution of C. (To illustrate with an example: Transmitter A may have made a greater contribution than transmitter B, because although both refused vaccinations, transmitter A made more reckless visits to hospitals and other environments in which immunosuppressed individuals reside. Transmitter $\mathrm{C}$ took the most stringent hygiene precautions of all, but $\mathrm{C}$ was also the blogger who spread 'antivaccination' ideas to $\mathrm{A}$ and $\mathrm{B}$, such that they would have been much more likely to get a vaccination had $\mathrm{C}$ not written the blog. We might think that C's contribution is so different from A's and B's that it cannot obviously be rated as greater, less than or equivalent in magnitude to either of them.) How much blame does $\mathrm{C}$ deserve relative to these others? Any particular answer will involve some arbitrariness.

At least one further question remains: does the blame deserved by one agent change, depending on whether other agents (or chancy processes) are involved in causing the harm?

\footnotetext{
${ }^{\mathrm{ix}}$ And following David Lewis, it could be argued that this practice does treat all wrongdoers equally: they are all subjected to equivalent risks of being blamed. ${ }^{24}$

${ }^{x}$ It also anticipates a potential objection in that we need not trace the causal chain back ad infinitum since only some members of the chain had reasonable opportunity to act otherwise: for example, when an outbreak in a high-income country begins with an imported case from a low-income country or a community with poor access to vaccination.
} 
Suppose that an agent's causal contribution to an outcome (including the degree to which he foresaw and intended the outcome) is the same in two scenarios S1 and S2, but that in S1 the outcome is facilitated (or hindered) by another agent, whereas in S2 he achieves the outcome alone. Does the agent deserve the same or a different degree of blame for the outcomes in these alternative scenarios? One possibility is to treat the total quantum of blame deserved for a given harm as constant, regardless of the number of agents, and to distribute it among the agents who causally contribute (eg, if three play similar roles in collectively causing a death, they may each deserve a reduced share of the blame that would ordinarily be ascribed to one individual for that death). This is what typically happens with liability to pay compensation. The amount of compensation required is constant, and mechanisms like joint and several liability allow that constant sum to be distributed in a variety of ways over the liable parties. On this approach, the presence of an additional agent will lead to a reduction in degree of blame deserved. Another possibility is that the blame deserved by the agent is identical in the two cases: despite the fact that our agent had an accomplice in his blameworthy deed, the degree of blame he deserves for the harm remains the same. ${ }^{\text {xi }}$

Regardless of how we resolve these issues, it should be stressed again that any such proposal that makes causation a necessary condition of blame for harm will be affected by moral luck (just as in the case of blaming the proximate cause T100). Given this, we may wish to consider whether it is preferable (in practice) to adopt other policies-such as vaccine mandateswhich would arguably involve a fairer distribution of risk and avoid the case where an unlucky subset of risk imposers would be blamed for their chance involvement in harms.

While it may not be possible to prescribe one uniquely preferred approach to apportioning moral responsibility to individuals in cases of collective responsibility, we believe that at least one of the above methods of attributing blame is defensible, and that is all we require for the defence of our thesis. While the incompleteness of our account may leave some degree of arbitrariness in the choice of rules to ascribe responsibility in theory and practice, this is no worse than the degree of arbitrariness in extant norms and laws: we punish attempted murderers less harshly than successful ones; we blame reckless drivers who cause injury much more harshly than those who luckily avoid injuring anyone; and we are of course resigned to blaming much less harshly those who simply evade detection.

\section{OBJECTION: VACCINATION IS SUPEREROGATORY}

The final objection we consider concedes that non-vaccinators are responsible for imposing risks on others, but denies that they are blameworthy. The idea is that while it might be praiseworthy to obtain a vaccination, it is strictly supererogatory to do so. Refusal to vaccinate is permissible either because vaccination comes at a significant cost to oneself or because the magnitude of risk imposed is not considered morally significant. This latter argument might proceed either by asserting that the absolute risk is small or by asserting that the overall risks and benefits of some social practice are equitably shared across the population.

${ }^{x i}$ Cf. Robert Nozick: "Responsibility is not a bucket in which less remains when some is apportioned out; there is not a fixed amount of punishment or responsibility which one uses up so that none is left over for the other." 25

\section{Partiality to self}

It is unreasonable to expect individuals to do everything they can to minimise risks to others. In particular, it is hard to accept that individuals have to bear significant risks of injury to themselves in order to achieve modest risk reductions for numerous others. Even if the net effect of the action is to reduce levels of expected harm to others, we might hold that-in some casesthere is a reasonable degree of partiality to oneself, such that it is merely supererogatory to undergo the risk in question. Serving as an experimental subject in a new drug trial might be an example where the risks are distributed in this fashion.

Having stated this objection, it is evidently implausible in the case of vaccination. First and foremost, non-vaccinators usually make themselves worse off by refusing a vaccine. So it is simply not the case that they would have to undergo a significant cost in order to reduce risk to others. But suppose we defer to a sincere non-vaccinator's claim that they believe it to be an undue hardship to receive a vaccine. How then do the burdens of vaccination compare to the potential risks to others? Taking measles as an example again, the risk of death due to vaccination is zero and the risk of serious vaccine reaction requiring hospitalisation is around $0.003 \%$ per dose, ${ }^{7}$ whereas the risk of death due to measles in an immunocompetent person is up to 1 in 350-1200 per infection and the risk of significant disease requiring hospitalisation is approximately $20 \% .^{3}$ Thus, in terms of hospitalisation, it is around 6000 times more dangerous to have measles than to have a dose of measles vaccine (and in terms of risk of death, the comparison is even more stark). Since the virus is so infectious, the risk of transmitting it while infected is high, and so the risk of harming or killing others is not negligible. For the risk of harming others to be so low as to be commensurate with the risk of vaccination, the lifetime odds of being part of a chain that reaches a non-immune person would have to be significantly lower than 6000 to 1 against (which could, admittedly, be the case if vaccination rates are sufficiently high and stable).

Second, even if it could be shown that the requisite probabilities are met in one place and at one point in time such cases will be exceedingly rare and difficult to identify. The risks change as a greater or lesser number of others opt out of vaccination, and as more people travel and cases are imported from other populations (which frequently occurs for many vaccinepreventable diseases).

Finally, non-vaccinators will tend to benefit from the herd immunity that is achieved by others undertaking vaccination, giving rise to a free-riding concern. ${ }^{26}$ Partiality to oneself is a much less plausible reason for failing to do good if one is already benefiting substantially from the equivalent sacrifices of others.

\section{Small risks}

Once herd protection is established at some level, the individual risk contributed by one non-vaccinator is admittedly small. It could be argued that it is therefore inappropriate to regard these risks as blameworthy. But this is, to use a term from Derek Parfit, a mistake in moral mathematics. ${ }^{27}$ Even small contributions are morally significant, especially (i) when small risks are run many times, as when the unvaccinated have social contact with many others, and (ii) where, taken together, they cause great harm. Herd protection is not binary-that is, it is not 'present' or 'absent', depending on whether some threshold rate of vaccination has been achieved. Herd protection is a probabilistic phenomenon reflecting a higher or lower level of the 
'background' risk of sustained transmission of infection. Unless or until an infectious disease has been eradicated worldwide, there is thus no level at which the risk of harming others by remaining unvaccinated is zero. Further, if the number of nonvaccinators is small, then although the total risk of harm to others will be small, each individual will be responsible for a sizeable proportion of that total risk.

\section{Distribution of risks}

In some cases, societies allow small risks to be imposed on others because these risks are part of a social system of mutual risk taking that, overall, produces significant benefits; driving personal automobiles may be one example. Yet, for such a system of shared risk to be justifiable, the risks and benefits must be shared equitably. ${ }^{28}$ Systems that allow non-medical exemptions for vaccination blatantly fail this requirement. Infants are vulnerable in multiple ways and many immunosuppressed people are already badly off in terms of health. The burden of risk will fall inequitably on these groups. Both the probability of infection and the probability of severe disease and death (upon infection) are higher for the vulnerable. By contrast, a system of mandatory vaccination (except for those excluded for medical reasons) involves sharing the small risk of vaccination equitably across the population, for significant health benefits, shared by all.

\section{ALTERNATIVE DUTY ASSIGNMENTS}

It might be wondered what concrete assignments of rights and obligations would be morally acceptable ways to address the risk of infectious diseases. In the absence of mandatory vaccination, should non-vaccinators be required to take other steps to reduce the risks they impose on others? Or should systems of liability hold all non-vaccinators to account for their risk imposition, whether or not specific harms arise $?^{20}$

An argument that the present assignment of obligations is inadequate can be made from considerations of economic efficiency. The risks imposed by non-vaccinators constitute a cost that is not consented to. In the jargon of economics, this is an externality: a cost that falls on people not participating in the relevant activity. And where there are externalities, there will often be inefficiency: people will choose a balance of activities that does not optimise welfare. ${ }^{29}$ In this case, the cost to the immunosuppressed of being exposed to a risk of infection is greater than the value to the unvaccinated of remaining unvaccinated. To identify how to remove the inefficiency, we can imagine various thought experiments, regarding what would happen if it were possible to negotiate a mutual agreement without excessive transaction costs, assuming a determinate division of rights.

On one salient alternative, imagine that the unvaccinated have a right to remain unvaccinated, but that the immunosuppressed can band together to purchase the compliance of the unvaccinated with a universal vaccination scheme. Given the very significant gains that the immunosuppressed stand to achieve, it is plausible that they would be willing to pay more than enough to obtain the compliance of at least a very large proportion of the unvaccinated community. The result would be to improve the position of the immunosuppressed, who are now better protected from immunity, and to improve the position of the unvaccinated, who have been adequately compensated for frustrating their preference for non-vaccination. This is a Pareto superior outcome, and the inefficiency has been removed. A scheme like this, on the other hand, could create perverse incentives for people to cultivate or imitate a preference not to be vaccinated.

Another salient possibility is to allocate the immunosuppressed an enforceable right not to be exposed to (avoidable) infection risks. In this case, the immunosuppressed could demand that those who chose not to be vaccinated (i) remain absent from public life, (ii) provide monetary compensation for the risks they impose on the immunosuppressed, (iii) undertake controlled infection ${ }^{\mathrm{xii}}$ or (iv) become vaccinated. It is very plausible that (iv) is the most preferred option for the nonvaccinators, given the very high costs of the other options. So, again, the inefficiency will have been removed: the immunosuppressed will enjoy the benefits of universal vaccination, and a scheme like this would not obviously create perverse incentives.

Both of these scenarios give very clear support to the intuitive claim that it is unreasonable for some to impose the risks of non-vaccination upon the immunosuppressed. It is unreasonable because it is possible to bring about other arrangements that make some better off, and nobody worse off.

Both of the above scenarios are admittedly unrealistic because it would be intolerably costly to engage in all the relevant transactions involving everyone with whom we might need to interact over the course of our lives. But a more realistic, centralised proposal is that those who refuse vaccination might merely be given the choice between (i) providing monetary compensation for the risks they impose on the immunosuppressed or (ii) becoming vaccinated. Monetary compensation could be achieved in practice via imposition of a new (non)vaccination tax. Those who are vaccinated could receive a rebate for the tax in question, so only those who remain unvaccinated end up paying. The revenue raised could be paid out in individual compensation settlements for infection-related harms or could simply be paid directly to vulnerable individuals as compensation for exposure to risk itself.

\section{CONCLUSIONS}

A typical person transmitting infection may seem innocent because they do not choose to contract or transmit the infection, just as the driver of a car rarely chooses the moment when his brakes fail. Yet we are all at risk of being both the victims and vectors of infectious disease, and by taking reasonable steps, where possible, to ensure that we are immune from being a victim, we also prevent ourselves from being a vector. ${ }^{23}$ Vaccination is a prime example of an action that, in this way, prevents harm to oneself as well as harm to others. We have argued that those who opt out of vaccination and transmit preventable disease to others can be considered morally responsible for the harms and deaths that result. Our current practices of attributing blame may not regard the avoidable transmission of vaccine-preventable disease as villainous behaviour, but the arguments of this article demonstrate that this attitude might warrant change.

Contributors All authors contributed to the research and the writing of this article, and approved the final manuscript.

Funding Green Templeton College, Ethox Centre and Green Templeton College, Oxford University (Andrew Markus Visiting Scholarship), Monash University (Australian Postgraduate Award).

Competing interests None declared.

\footnotetext{
${ }^{x i i}$ Controlled infection would aim to make the person immune and thus pose no risk of transmitting the disease. This would involve intentional infection with medical oversight in an isolated environment where transmission is prevented. Medical oversight decreases risks to the infected individual, but it is worth noting that for many vaccine-preventable diseases there is no curative treatment for most complications, should they arise, which would limit the application of this approach (and demonstrates another advantage of vaccination).
} 
Provenance and peer review Not commissioned; externally peer reviewed.

Open Access This is an Open Access article distributed in accordance with the Creative Commons Attribution Non Commercial (CC BY-NC 4.0) license, which permits others to distribute, remix, adapt, build upon this work non-commercially, and license their derivative works on different terms, provided the original work is properly cited and the use is non-commercial. See: http://creativecommons.org/ licenses/by-nc/4.0/

\section{REFERENCES}

1 Roush SW, Murphy TV, Vaccine-Preventable Disease Table Working G. Historical comparisons of morbidity and mortality for vaccine-preventable diseases in the United States. JAMA 2007;298:2155-63.

2 Halsey NA, Salmon DA. Measles at Disneyland, a problem for all ages. Ann Intern Med 2015;162:655-6.

3 Orenstein WA, Perry RT, Halsey NA. The clinical significance of measles: a review. J Infect Dis 2004;189(Suppl 1):S4-16.

4 Baxi R, Mytton OT, Abid M, et al. Outbreak report: nosocomial transmission of measles through an unvaccinated healthcare worker-implications for public health. J Public Health (Oxf) 2014;36:375-81.

5 Simons E, Ferrari M, Fricks J, et al. Assessment of the 2010 global measles mortality reduction goal: results from a model of surveillance data. Lancet 2012;379:2173-8.

6 Paul Y. Herd immunity and herd protection. Vaccine 2004;22:301-2.

7 Patja A, Davidkin I, Kurki T, et al. Serious adverse events after measles-mumpsrubella vaccination during a fourteen-year prospective follow-up. Pediatr Infect Dis $J$ 2000;19:1127-34.

8 Kunisaki KM, Janoff EN. Influenza in immunosuppressed populations: a review of infection frequency, morbidity, mortality, and vaccine responses. Lancet Infect Dis 2009;9:493-504.

9 Glück T, Müller-Ladner U. Vaccination in patients with chronic rheumatic or autoimmune diseases. Clin Infect Dis 2008;46:1459-65.

10 Fine P, Eames K, Heymann DL. "Herd immunity": a rough guide. Clin Infect Dis 2011;52:911-16.
11 Fox JP, Elveback L, Scott W, et al. Herd immunity: basic concept and relevance to public health immunization practices. Am J Epidemiol 1971;94:179-89.

12 Rashid H, Khandaker G, Booy R. Vaccination and herd immunity: what more do we know? Curr Opin Infect Dis 2012;25:243-9.

13 Dawson $A$. Herd protection as a public good: vaccination and our obligations to others. In: Dawson A, Verweij M, eds. Ethics, prevention and public health. Oxford University Press, Oxford, UK 2009:160-78.

14 Dawson A. Vaccination ethics. Public Health Ethics 2011:143-53. http://dx.doi.org/ 10.1017/CB09780511862670.009

15 Verweij $M$, Dawson A. Ethical principles for collective immunisation programmes. Vaccine 2004;22:3122-6.

16 Verweij M. Obligatory precautions against infection. Bioethics 2005;19:323-35.

17 Harris J, Holm S. Is there a moral obligation not to infect others? BMJ 1995;311:1215-17.

18 Rachels J. Killing and letting die. In: Becker LC, Becker M, Becker C, eds. Encyclopedia of ethics. 2nd edn. Routledge, 2001;2:947-50.

19 Handfield T, Pisciotta T. Is the risk-liability theory compatible with negligence law? Legal Theory 2005;11:387-404.

20 McCarthy D. Liability and risk. Philos Public Affairs 1996;25:238-62.

21 Williams BAO, Nagel T. Moral luck. Proc Aristotelian Soc Supplementary Volumes 1976;50:115-51.

22 Smiley M. Collective responsibility. In: Zalta EN, ed. The Stanford encyclopedia of philosophy. Fall, 2011.

23 Battin M, Francis L, Jacobsen J, et al. The patient as victim and vector. New York, NY: Oxford Press, 2007:90-2.

24 Lewis D. The punishment that leaves something to chance. Philos Public Affairs 1989:53-67.

25 Nozick R. Anarchy, state, and utopia. Basic Books, 1974.

26 Selgelid MJ. Ethics of infectious disease control. In: Heggenhougen HK, ed. International encyclopedia of public health. Oxford: Academic Press, 2008:486-93.

27 Parfit D. Reasons and persons. Oxford University Press, 1984.

28 Hansson SO. Ethical criteria of risk acceptance. Erkenntnis 2003;59:291-309.

29 Coase RH. The problem of social cost. J Law Econ 1960;3:1-44. 